\title{
Continuous Sample Paths in Quantum Field Theory
}

\author{
John T. Cannon \\ Rockefeller University, New York, N.Y., USA
}

Received August 20, 1973

\begin{abstract}
Nelson's free Markoff field on $\mathbb{R}^{l+1}$ is a natural generalization of the Ornstein-Uhlenbeck process on $\mathbb{R}^{1}$, mapping a class of distributions $\phi(x, t)$ on $\mathbb{R}^{l} \times \mathbb{R}^{1}$ to mean zero Gaussian random variables $\phi$ with covariance given by the inner product $\left(\left(m^{2}-\Delta-\frac{\partial^{2}}{\partial t^{2}}\right)^{-1} \cdot, \cdot\right)_{2}$. The random variables $\phi$ can be considered functions $\phi\langle q\rangle$ $=\int \phi(\boldsymbol{x}, t) q(\boldsymbol{x}, t) d \boldsymbol{x} d t$ on a space of functions $q(\boldsymbol{x}, t)$. In the O.U. case, $l=0$, the classical Wiener theorem asserts that the underlying measure space can be taken as the space of continuous paths $t \rightarrow q(t)$. We find analogues of this, in the cases $l>0$, which assert that the underlying measure space of the random variables $\phi$ which have support in a bounded region of $\mathbb{R}^{l+1}$ can be taken as a space of continuous paths $t \rightarrow q(\cdot, t)$ taking values in certain Soboleff spaces.
\end{abstract}

\section{Introduction}

The Feynman-Kac formula, which solves the Schrödinger equation by giving its imaginary time Green's function as an integral over Wiener space, has an infinite-number-of-degrees-of-freedom analogue which solves regularized Boson quantum field theories by giving their Schwinger function as integrals over the probability space associated with the free Markoff field (cf. [1-4]). Detailed structure of the associated probability space is not needed to obtain the basic formulae; but Wiener's theorem on the continuity of sample paths is of well known usefulness in the case of a finite number of degrees of freedom. Here we shall attempt to find its analogue in the infinite case.

The free Markoff field was introduced by Nelson [5] who used only an abstract representation of the underlying probability space but indexed the Gaussian random variables by the elements of the Hilbert space $\mathfrak{G}$ of real distributions on $\mathbb{R}^{l+1}$ in the norm $\left\|\left(m^{2}-\Delta\right)^{-1 / 2} \cdot\right\|_{2}$, where $\Delta$ is the Laplacian on $\mathbb{R}^{l+1}$. That is, each element of the Hilbert space was made a Gaussian random variable of mean zero so that the covariance of two elements would be their inner product. We will take the dual point of view and begin with the underlying "probability space" as the Hilbert space $\mathfrak{Q}$ of real functions in the norm $\left\|\left(m^{2}-\Delta\right)^{1 / 2} \cdot\right\|_{2}$ 
in the isonormal distribution. (Basic facts about the isonormal distribution will be reviewed in $\S 1$.) An element $\phi \in \mathfrak{H}$ is a function on $\mathfrak{Q}$ taking the value

$$
\phi\langle q\rangle=\int_{\mathbb{R}^{l+1}} \phi(x) q(x) d x
$$

at $q \in \mathfrak{Q}$. Under the isonormal distribution on $\mathfrak{Q}$, the $\phi \in \mathfrak{H}$ are the appropriate random variables for the Markoff field. But the measure defined by the isonormal distribution is countably additive only on appropriate extensions of the Hilbert space $\mathfrak{Q}$. For example, if $A$ is a positive Hilbert-Schmidt operator, it suffices to complete $\mathfrak{Q}$ in the norm $\left\|\left(m^{2}-\Delta\right)^{1 / 2} A \cdot\right\|_{2}$ (cf. [6-8]). In particular, the measure is countably additive on the space of tempered distributions, which is the representation used by Dimock and Glimm [9]. Our problem is to find minimal extensions of $\mathfrak{Q}$ with natural properties.

The isonormal distribution on an abstract Hilbert space has been studied by Segal, Friedrichs, and others (cf. [10]) and, in particular, the problem of attaining countable additivity by extension has been solved by Gross [8] whose results we will review in Theorem 1.5. Gross defined a measurable norm on the Hilbert space to be a norm having small probability of being large (under the isonormal distribution) on the orthogonal complement of a finite dimensional subspace and proved that the isonormal distribution defines a (countably additive) probability measure on (the Borel field of) the completion of the Hilbert space with respect to a measurable norm. From this point of view, the classical Wiener theorem asserts that the supremum norm is measurable on the Hilbert space of real functions on $[0, T]$, that are zero at the origin, in the norm $\left\|\frac{d}{d t} \cdot\right\|_{2}$.

Let $\mathscr{R}=\boldsymbol{R}_{0} \times(0, T)$ be a rectangle in $\mathbb{R}^{l+1}$ and let $\mathfrak{Q}(\mathscr{R})$ be the completion of $\mathscr{C}_{0}^{\infty}(\mathscr{R})$ in $\left\|\left(m^{2}-\Delta\right)^{1 / 2} \cdot\right\|_{2}$. Let $\Delta_{0}$ be the Dirichelet Laplacian on $\boldsymbol{R}_{0}$ and let $\left\{\phi_{\alpha}\right\}$ be an orthogonal basis in $\mathscr{L}^{2}\left(\boldsymbol{R}_{0}\right)$ of eigenvectors of $\left(m^{2}-\Delta_{0}\right)$ with corresponding eigenvalues $\left\{m_{\alpha}^{2}\right\}$. Write $q_{\alpha}(t)=\int_{\boldsymbol{R}_{0}} \phi_{\alpha} q(\cdot, t)$. Our central result, Theorem 3.1, is that the norms

and

$$
\sup _{0 \leqq t \leqq T}\|q(\cdot, t)\|_{p, \gamma} \equiv\left\{\begin{array}{l}
\sup _{0 \leqq t \leqq T}\left(\sum_{\alpha} m_{\alpha}^{-\gamma p}\left|q_{\alpha}(t)\right|^{p}\right)^{1 / p} \\
\sup _{t, \alpha} m_{\alpha}^{-\gamma}\left|q_{\alpha}(t)\right|, \quad \text { if } p=\infty
\end{array}\right.
$$

$$
\sup _{0 \leqq t \leqq T}\left\|\left(m^{2}-\Delta_{0}\right)^{-\frac{\gamma^{\prime}}{2}} q(\cdot, t)\right\|_{p^{\prime}}
$$


are measurable on $\mathfrak{Q}(\mathscr{R})$ provided $1 \leqq p \leqq \infty, \gamma>\frac{l}{p}-\frac{1}{2}$, and $2 \leqq p^{\prime} \leqq \infty$, $\gamma^{\prime}>l\left(1-\frac{1}{p^{\prime}}\right)-\frac{1}{2}$. Let $\mathfrak{Q}^{p, \gamma}(\mathscr{R})$ denote the completion of $\mathfrak{Q}(\mathscr{R})$ in the norm (0.2). The norm (0.3) is dominated by the norm $(0.2)$ with $p=\left(1-\frac{1}{p^{\prime}}\right)^{-1}, \gamma^{\prime}=\gamma$, by the Hausdorff, Young, and F. Riesz theorem; so we won't consider it explicitly.) If $q \in \mathfrak{Q}^{p, \gamma}(\mathscr{R})$, then

$$
t \mapsto q(\cdot, t)
$$

is a continuous sample path in the norm $\|\cdot\|_{p, \gamma}$.

So the isonormal distribution on $\mathfrak{Q}(\mathscr{R})$ defines a probability measure $\mu_{0}$ on $\mathfrak{Q}^{p, \gamma}(\mathscr{R})$. Evaluating $\phi \in \mathfrak{H}$ as in $(0.1),\left\{\mu_{0}, \mathfrak{Q}^{p, \gamma}(\mathscr{R})\right\}$ is an underlying probability space for the Dirichelet free Markoff field, not the free Markoff field. However, in Proposition 2.1 we prove that the two Markoff fields are locally equivalent (in the sense of absolute continuity). Thus we obtain in Corollary 3.3 the following local result for the free Markoff field: If $\mathscr{R}_{1}$ is appropriately contained in $\mathscr{R}$ there is a measure $\mu$ which is absolutely continuous with respect to $\mu_{0}$ so that $\left\{\mu, \mathfrak{Q}^{p, \gamma}(\mathscr{R})\right\}$ is an underlying probability space for the free field random variables $\phi \in \mathfrak{H}$ with supp $\phi \subset \mathscr{R}_{1}$.

To illustrate the use of $\left\{\mu, \mathfrak{Q}^{p, \gamma}(\mathscr{R})\right\}$, consider a positive polynomial $P$ and fix an open rectangle $I \times(0, t) \subset \mathscr{R}_{1}$ with $\mathscr{R}_{1}$ appropriately contained in $\mathscr{R}$; let $F_{\kappa}(\xi)$ be one or zero for $\xi \leqq \kappa$ or $\xi>\kappa$, respectively, and define $q_{\kappa}(\cdot, t)=F_{\kappa}\left(\left(m^{2}-\Delta_{0}\right)\right) q(\cdot, t) \quad$ for $\quad q \in \mathfrak{Q}^{p, \gamma}(\mathscr{R})$. Let $V_{\kappa}\langle q(\cdot, t)\rangle$ $=\int_{\boldsymbol{I}} P\left(q_{\kappa}(\boldsymbol{x}, t)\right) d \boldsymbol{x}$. Then $q \mapsto \sum_{j=1}^{n} \frac{t}{n} V_{\kappa}\left\langle q\left(\cdot, \frac{j t}{n}\right)\right\rangle$

and

$$
q \mapsto V_{\kappa}\langle q\rangle \equiv \int_{0}^{t} V_{\kappa}\langle q(\cdot, t)\rangle d t
$$

are continuous functions on $\mathfrak{Q}^{1, \gamma}, \gamma>l-\frac{1}{2}$, by the Hausdorff, Young, and F. Riesz theorem. By the continuity of (0.4), the first converges pointwise to the second as $n \rightarrow \infty$. So by the Lebesgue dominated convergence theorem

$$
\exp \left\{-\sum_{j=1}^{n} \frac{\mathrm{t}}{n} V_{\kappa}\left\langle q\left(\cdot, \frac{j t}{n}\right)\right\rangle\right\} \stackrel{\mathscr{L}^{p}}{\longrightarrow} \exp \left\{-V_{\kappa}\langle q\rangle\right\}
$$

in $\mathscr{L}^{p}\left(\mu, \mathfrak{Q}^{1, \gamma}(\mathscr{R})\right), 1 \leqq p<\infty$, as $n \rightarrow \infty$. If $\{\mu, \overline{\mathbb{Q}}\}$ is any representation of the probability space underlying the (global) free Markoff field, then the convergence $(0.7)$ must also hold in $\mathscr{L}^{p}(\mu, \overline{\mathfrak{Q}})$.

Of course, $V_{\kappa}$ in (0.7) can be replaced by the Wick ordered function $: V_{\kappa}:$. Then for the case $l=1$, as is well known, we also have the convergence

$$
\exp \left\{-: V_{\kappa}:\right\} \stackrel{\mathscr{L}^{p}}{\longrightarrow} \exp \{-: V:\}
$$


in $\mathscr{L}^{p}(\mu, \overline{\mathfrak{Q}}), 1 \leqq p<\infty$, as $\kappa \rightarrow \infty$. In fact, it is elementary that $: V_{\kappa}: \rightarrow: V:$ in $\mathscr{L}^{2}$ and hence in probability. Therefore we have the convergence (0.8) in probability. By the Nelson-Glimm semi-boundedness argument [11] the measures $\exp \left\{-p: V_{\kappa}:\right\} d \mu$ are uniformly absolutely continuous with respect to $d \mu$ and they are equicontinuous from above at the empty set. Therefore we have the $\mathscr{L}^{p}$ convergence of $(0.8)$ by a generalization of the dominated convergence theorem [12; p. 108]. (The original argument is due to Rosen [13].)

Let $\mathscr{F}_{t}$ be the subspace of $\mathscr{L}^{2}(\mu, \overline{\mathfrak{Q}})$ generated by

$$
\left\{\phi \in \mathfrak{H}, \operatorname{supp} \phi \subset \mathbb{R}^{l} \times\{t\}\right\} .
$$

If $f \in \mathscr{F}_{0}$ and $g \in \mathscr{F}_{t}$, then it follows from Nelson's hypercontractivity theorem [5] that

$$
\|f g\|_{r} \leqq\|f\|_{2} \cdot\|g\|_{2}, \quad r=2\left(1+e^{-m|t|}\right)^{-1} .
$$

The time-zero Boson quantum free field $\Phi(\phi)$ can be identified with multiplication by $\phi \in \mathscr{F}_{t}$ as an operator in $\mathscr{F}_{t} \oplus i \mathscr{F}_{t}$ [5]. The relevance of (0.7) and (0.8) for quantum field theory can be seen by setting $p=\left(1-\frac{1}{r}\right)^{-1}$ and using Hölder's inequality with (0.9).

and let

$$
h\langle q\rangle=\frac{\exp \{-: V:\langle q\rangle\}}{\int_{\mathbb{Q}} \exp \{-: V:\} d \mu}
$$

$$
h_{\mathscr{R}_{1}}\langle q\rangle=\mathscr{E}_{\mu}\left(h \mid \phi \in \mathfrak{H}, \operatorname{supp} \phi \subset \mathscr{R}_{1}\right)
$$

be the conditional expectation of $h$ with respect to $\mu$ and the $\sigma$-field generated by $\left\{\phi \in \mathfrak{H}\right.$, supp $\left.\phi \subset \mathscr{R}_{1}\right\}$, with $: V:\langle q\rangle=\int_{I_{2}} d t \int_{I_{1}} d x: P(q(x, t))$ :. So $h$ and $h_{R_{1}}$ depend on the rectangle $I_{1} \times I_{2}$. We no longer relate the rectangle $I_{1} \times I_{2}$ to $\mathscr{R}_{1}$ (earlier we were concerned with the case $I_{1} \times I_{2}\left(\mathscr{R}_{1}\right)$. But suppose again that $\mathscr{R}_{1}$ is appropriately contained in $\mathscr{R}$. We have the result that $\left\{h_{\mathscr{R}_{1}} d \mu, \mathbb{Q}^{p, \gamma}(\mathscr{R})\right\}$, with $p$ and $\gamma$ as before, is a representation of the underlying probability space for the random variables $\phi \in \mathfrak{H}$ with supp $\phi \subset \mathscr{R}_{1}$ in the expectation $\int_{0}(\cdot) h d \mu$. That is, the same continuous sample paths $t \rightarrow q(\cdot, t)$ suffice for the non-free theory with interaction $: V:$. Furthermore, this is true also in the limit $I_{1} \times I_{2} \rightarrow \mathbb{R}^{2}$, at least in the case of $m$ sufficiently large. In fact, Newman [14] has shown that the measures $h_{\mathscr{R}_{1}} d \mu$ converge in this limit, for $m$ large, to a probability measure which is again absolutely continuous with respect to $\mu$.

To obtain some idea of the sharpness of our result, consider the function

$$
q \mapsto \int_{\boldsymbol{R}_{0}}\left(\left(m^{2}-\Delta_{0}\right)^{-\frac{\gamma}{2}} q(\boldsymbol{x}, t)\right)^{2} d \boldsymbol{x}
$$


for fixed $t \in(0, T), \gamma>\frac{l-1}{2}$. It is continuous on $\mathfrak{Q}^{2, \gamma}(\mathscr{R})$ and it differs from its Wick ordered version by a constant proportional to $\sum_{\alpha} m_{\alpha}^{-1-2 \gamma}<\infty$. As $\gamma \rightarrow \frac{l-1}{2}$, the function $(0.12)$ converges to a continuous function on $\mathfrak{Q}^{2, \frac{l-1}{2}}(\mathscr{R})$, the Wick ordered version converges in $\mathscr{L}^{2}$, and their difference diverges to infinity. Therefore, $\mathfrak{Q}^{2, \frac{l-1}{2}}(\mathscr{R})$ has measure zero in $\left\{\mu_{0}, \mathfrak{Q}^{p, \gamma}(\mathscr{R})\right\}$. So the condition $\gamma>\frac{l}{p}-\frac{1}{2}$ is sharp in the case $p=2$. On the other hand, it doesn't seem to be obviously natural if $p \neq 2$.

After preparing this paper we heard the lectures of M.Reed at the "International School of Mathematical Physics at Erice-Sicily; First Course on Constructive Quantum Field Theory; 1973" (which will appear in proceedings, eds. G. Velo and A.S. Wightman, pub. SpringerVerlag) in which related material was discussed. In particular, we note a result of 0 . Lanford that the paths $q$ are a.s. too rough to be measures $q(\boldsymbol{x}, t) d \boldsymbol{x} d t$ in some region of $\mathbb{R}^{l+1}$. Therefore the condition $p^{\prime} \geqq 2$ for the measurability of the norm $(0.3)$ is necessary in the case $l=1$, i.e. we cannot drop this condition to obtain measurability of $\sup _{0 \leqq t \leqq T}\|q(\cdot, t)\|_{p^{\prime}}$ for $p^{\prime}<2$.

We will assume that the mass $m$ is positive. However, this assumption plays no role in statements that concern the Dirichelet field only. In particular, Theorem 3.1 and its first corollary hold for $m=0$.

\section{§ 1. Notation and Generalities on Gaussian Random Variables [6 - 10]}

Let $\mathfrak{H}, \mathfrak{Q}$ denote a dual pair of real seperable Hilbert spaces. An element $\phi \in \mathfrak{H}$ as a linear function on $\mathfrak{Q}$ will be denoted $\phi\langle\cdot\rangle$. Duality will be notated as in $A^{\dagger} \phi\langle q\rangle=\phi\langle A q\rangle$. The Riesz identification of $\mathfrak{H}$ and $\mathfrak{Q}$ will be notated as in $\hat{\mathfrak{H}}=\mathfrak{Q}, \hat{\mathfrak{Q}}=\mathfrak{H},(\phi, \hat{q})_{\mathfrak{H}}=\phi\langle q\rangle=(\hat{\phi}, q)_{\mathfrak{Q}}$. A complex valued function $f\langle\cdot\rangle$ on $\mathbb{Q}$ is called tame if there is a finite set $\left\{\phi_{1}, \ldots, \phi_{n}\right\}$ of elements of $\mathfrak{H}$ and a Borel function $\tilde{f}$ on $\mathbb{R}^{n}$ such that

$$
f\langle q\rangle=\tilde{f}\left(\phi_{1}\langle q\rangle, \phi_{2}\langle q\rangle, \ldots, \phi_{n}\langle q\rangle\right), \quad q \in \mathfrak{Q} .
$$

If $\mathfrak{H}_{1}$ is a subspace of $\mathfrak{H}$ let $\mathscr{T}\left(\mathfrak{H}_{1}\right)$ denote the space of tame functions on $\mathfrak{Q}$ which can be written in the form (1.1) with all $\phi_{i} \in \mathfrak{H}_{1}$. If $T$ is a bounded linear operator on $\mathfrak{Q}$ which is symmetric, positive and invertible with $T^{-1}$ bounded, we define the normal expectation of $f \in \mathscr{T}(\mathfrak{H})$ relative to $T$ as

$$
\langle\mathfrak{Q}, T \mid f\rangle=\pi^{-n / 2} \int_{-\infty}^{\infty} \ldots \int_{-\infty}^{\infty} \tilde{f}\left(x_{1}, \ldots, x_{n}\right) e^{-\sum_{i=1}^{n} x_{i}^{2}} d x_{1} \ldots d x_{n}
$$


if $\tilde{f}$ and $\phi_{i}$ in (1.1) are chosen so that the $\phi_{i}$ are orthonormal in the inner product dual to $(T \cdot, \cdot)_{\mathfrak{Q}}$, namely $\left(T^{-1 \dagger} \cdot, \cdot\right)_{\mathfrak{H}}$. More generally, we define for $q_{1} \in \mathfrak{Q}$

$$
\left\langle\mathfrak{Q}, T, q_{1} \mid f\right\rangle=\left\langle\mathfrak{Q}, T \mid f_{1}\right\rangle, \quad f_{1}\langle q\rangle=f\left\langle q_{1}+q\right\rangle .
$$

If $\mathfrak{Q}_{1}$ is a subspace of $\mathfrak{Q}$ and $q_{1} \in \mathfrak{Q}$, we define the expectation on $\mathscr{T}(\mathfrak{H})$

$$
\begin{aligned}
\left\langle\mathfrak{Q}, \mathfrak{Q}_{1}, q_{1} \mid f\right\rangle & =\left\langle\mathfrak{Q}, I \mid f_{1}\right\rangle, \\
f_{1}\langle q\rangle & =f\left\langle q_{1}+P_{\mathfrak{Q}_{1}} q\right\rangle
\end{aligned}
$$

where $P_{\mathbb{Q}_{1}}$ is the orthogonal projection in $\mathfrak{Q}$ to $\mathfrak{Q}_{1}$. Note that

$$
\left.\begin{array}{rl}
\left\langle\mathfrak{Q}, T, q_{1} \mid e^{i \phi}\right\rangle & =e^{-i \phi\left\langle q_{1}\right\rangle} e^{-\frac{1}{4}\left(T^{-1 \dagger} \phi, \phi\right)_{\mathfrak{5}}} \\
\left\langle\mathfrak{Q}, \mathfrak{Q}_{1}, q_{1} \mid e^{i \phi}\right\rangle & =e^{-i \phi\left\langle q_{1}\right\rangle} e^{-\frac{1}{4}\left\|P_{\mathfrak{Q}_{1}}^{\dagger} \phi\right\|_{\mathfrak{5}}^{2}} .
\end{array}\right\}
$$

That is, the elements of $\mathfrak{H}$ are Gaussian random variables in the expectations (1.3) and (1.4) of mean $-\phi\left\langle q_{1}\right\rangle$ and respective covariances $2\left(T^{-1 \dagger} \phi_{1}, \phi_{2}\right)_{\mathfrak{H}}$ and $2\left(P_{\mathfrak{Q}_{1}}^{\dagger} \phi_{1}, \phi_{2}\right)_{\mathfrak{H}}$.

If $q_{1} \perp \mathbb{Q}_{1}$ in $\mathfrak{Q}$, then $\left\langle\mathbb{Q}, \mathbb{Q}_{1}, q_{1} \mid \cdot\right\rangle$ is the $\langle Q \mid \cdot\rangle$-conditional expectation relative to the affine subspace $q_{1}+\mathfrak{Q}_{1}$. Furthermore, $\left\langle\mathfrak{Q}, \mathfrak{Q}_{1}, \cdot \mid f\right\rangle$ for $f \in \mathscr{T}(\mathfrak{H})$ is a tame function on $\mathfrak{Q}_{1}^{\perp}$ and

$$
\left\langle\mathfrak{Q}_{1}^{\perp} \mid\left\langle\mathfrak{Q}, \mathfrak{Q}_{1}, \mid f\right\rangle\right\rangle=\langle\mathfrak{Q} \mid f\rangle \text {. }
$$

(We are writing $\langle\mathfrak{Q} \mid \cdot\rangle$ for $\langle\mathfrak{Q}, I \mid \cdot\rangle$, etc.)

An expectation on $\mathscr{T}(\mathfrak{H})$ can be represented by a probability measure, meaning that $\mathscr{T}(\mathfrak{H})$ can be represented by measurable functions on a measure space so that the expectation is given by the integral with respect to the stated probability measure. If two expectations can be represented by probability measures on the same measure space with the same representation of $\mathscr{T}(\mathfrak{H})$, they are called equivalent if the measures are equivalent in the sense of absolute continuity. Finally, given two equivalent expectations on $\mathscr{T}(\mathfrak{H})$ and a representation of one by a probability measure, the other is represented by an equivalent probability measure.

Lemma 1.1 (cf. [6-8]). If $T$ has the properties stated for (1.2) and in addition $(I-T)$ is Hilbert-Schmidt in $\mathbb{Q}$, then for $q_{1} \in \mathbb{Q},\langle\mathbb{Q} \mid \cdot\rangle$ and $\left\langle\mathfrak{Q}, T, q_{1} \mid \cdot\right\rangle$ are equivalent.

We will be concerned with the following consequence.

Lemma 1.2. Let $\mathfrak{H}_{1}$ be a subspace of $\mathfrak{H}$. The restriction of $\langle\mathfrak{Q} \mid \cdot\rangle$ to $\mathscr{T}\left(\mathfrak{H}_{1}\right)$ is equivalent to the restriction of $\left\langle\mathfrak{Q}, \mathfrak{Q}_{1}, q_{1} \mid \cdot\right\rangle$ to $\mathscr{T}\left(\mathfrak{H}_{1}\right)$ for any $q_{1} \in \mathbb{Q}$ and any subspace $\mathfrak{Q}_{1}$ of $\mathfrak{Q}$ such that

$$
\left.\begin{array}{l}
\left\|P_{\mathfrak{H}_{1}} P_{\hat{\mathfrak{Q}}_{1}^{\perp}} P_{\mathfrak{H}_{1}}\right\|<1, \\
P_{\mathfrak{H}_{1}} P_{\hat{\mathfrak{Q}}_{1}^{\perp}} P_{\mathfrak{H}_{1}} \quad \text { Hilbert-Schmidt in } \mathfrak{H} .
\end{array}\right\}
$$


Proof. We can identify $\mathscr{T}\left(\mathfrak{H}_{1}\right) \subset \mathscr{T}(\mathfrak{H})$ with the tame functions on $\hat{\mathfrak{H}}_{1}: f\langle q\rangle=f\left\langle P_{\hat{\mathfrak{H}}_{1}} q\right\rangle$. Then for $f \in \mathscr{T}\left(\mathfrak{S}_{1}\right)$,

$$
\left\langle\mathfrak{Q}, \mathfrak{Q}_{1}, q_{1} \mid f\right\rangle=\left\langle\hat{\mathfrak{H}}_{1},\left(P_{\hat{\mathfrak{H}}_{1}} P_{\mathfrak{Q}_{1}}\right)^{-1}, P_{\hat{\mathfrak{H}}_{1}} q_{1} \mid f\right\rangle
$$

provided $P_{\hat{\mathfrak{H}}_{1}} P_{\mathfrak{Q}_{1}}$ as an operator on $\hat{\mathfrak{G}}_{1}$ has an inverse. To see this, look at the formulas (1.5). So we are concerned with the equivalence of $\left\langle\hat{\mathfrak{H}}_{1} \mid \cdot\right\rangle$ with $\left\langle\hat{\mathfrak{H}}_{1},\left(P_{\hat{\mathfrak{H}}_{1}} P_{\mathfrak{Q}_{1}}\right)^{-1}, P_{\hat{\mathfrak{H}}_{1}} q_{1} \mid \cdot\right\rangle$. By the inequality $(1.7)$ there is a constant $c>0$ so that $\left\|P_{Q_{1}^{\frac{1}{1}}} \hat{\phi}\right\|_{Q} \leqq \sqrt{1-c^{2}}$ for all $\hat{\phi} \in \hat{\mathfrak{H}}_{1}$ with $\|\hat{\phi}\|_{\mathbb{Q}}=1$; so $\left\|P_{Q_{1}} \hat{\phi}\right\|_{Q}$ $\geqq c$ which implies that $\left\|P_{\hat{\mathfrak{H}}_{1}} P_{\mathbb{Q}_{1}} \hat{\phi}\right\|_{\mathbb{Q}} \geqq c^{2}>0$. This implies the desired invertibility. Now to apply Lemma 1.1 , we need to know that, in $\hat{\mathfrak{H}}_{1}$, $\left(I-\left(P_{\hat{\mathfrak{G}}_{1}} P_{\mathfrak{Q}_{1}}\right)^{-1}\right)$ is Hilbert-Schmidt or equivalently that $\left(I-P_{\hat{\mathfrak{G}}_{1}} P_{\mathfrak{Q}_{1}}\right)$ $=P_{\hat{\mathfrak{H}}_{1}} P_{\mathfrak{Q}_{1}^{\frac{1}{1}}} P_{\hat{\mathfrak{S}}_{1}}$ is Hilbert-Schmidt, which it is, being dual to the operator considered in (1.7).

Lemma 1.3. Let $\mathfrak{H}_{1}$ and $\mathfrak{Q}_{1}$ be respective subspaces of $\mathfrak{H}$ and $\mathfrak{Q}$ satisfying the conditions (1.7). Let $\mathfrak{H}_{2}$ be a subspace of $\mathfrak{H}_{1}$ and $\mathfrak{Q}_{2}$ a subspace of $\mathfrak{Q}$ that contains $\mathfrak{Q}_{1}: \mathfrak{H}_{2} \subset \mathfrak{S}_{1}, \mathfrak{Q}_{2} \supset \mathfrak{Q}_{1}$. Then $\mathfrak{H}_{2}$ and $\mathfrak{Q}_{2}$ satisfy the conditions (1.7).

Proof. Use the fact that, for $\phi \in \mathfrak{H}_{2}$,

$$
\left\|P_{\mathfrak{S}_{2}} P_{\mathfrak{Q}_{2}^{\frac{1}{2}}} \phi\right\| \leqq\left\|P_{\mathfrak{S}_{1}} P_{\mathfrak{Q}_{1}^{\frac{1}{1}}} \phi\right\|,
$$

and the fact that an operator $A$ is Hilbert-Schmidt if $\Sigma\left\|A \phi_{i}\right\|^{2}$ converges for some orthogonal basis $\left\{\phi_{i}\right\}$ and only if it converges for every orthonormal basis $\left\{\phi_{i}\right\}$.

If $\overline{\mathfrak{Q}}$ denotes a locally convex linear space in which $\mathfrak{Q}$ is dense with dual $\overline{\mathfrak{H}}$ dense in $\mathfrak{H}$, let $\mathscr{T}(\overline{\mathfrak{H}}) \subset \mathscr{T}(\mathfrak{H})$ denote the tame functions on $\overline{\mathfrak{Q}}$ : if $f \in \mathscr{T}(\mathfrak{H})$ then $f \in \mathscr{T}(\overline{\mathfrak{H}})$ if it can be put in the form (1.1) with the $\phi_{i} \in \overline{\mathfrak{H}}$. An expectation on $\mathscr{T}(\mathfrak{G})$ restricts to an expectation on $\mathscr{T}(\overline{\mathfrak{G}})$. A cylinder set in $\mathfrak{Q}$ or $\overline{\mathfrak{Q}}$ is a subset whose characteristic function belongs to $\mathscr{T}(\mathfrak{H})$ or $\mathscr{T}(\overline{\mathfrak{H}})$, respectively. Let $R(\mathfrak{Q})$ and $R(\overline{\mathfrak{Q}})$ denote the respective rings of cylinder sets. Corresponding to an expectation on $\mathscr{T}(\mathfrak{H})$ there is a probability defined on $R(\mathfrak{Q})$ and $R(\overline{\mathfrak{Q}})$ : Prob $\{A\}$ is the expectation of the characteristic function of $A$. We are concerned with the problem of choosing $\overline{\mathfrak{Q}}$ so that $\operatorname{Prob}\{\}$ defined from $\langle\mathfrak{Q} \mid \cdot\rangle$ is countably additive on $R(\overline{\mathbf{Q}})$ :

Definition (Gross [8, 15]). A norm $\|\cdot\| \|$ on $\mathfrak{Q}$ is called measurable if for every $a>0$ there exists a finite dimensional subspace $\mathfrak{Q}_{a}$ of $\mathfrak{Q}$ such that for every finite dimensional orthogonal projection $P$ in $\mathbb{Q}_{a}^{\perp}$,

$$
\operatorname{Prob}\{\|P q\| \mid>a\}<a,
$$

where $\operatorname{Prob}\{\cdot\}$ is defined from $\langle\mathfrak{Q} \mid \cdot\rangle$.

Lemma 1.4. (Gross [15]). A measurable norm on $\mathfrak{Q}$ is bounded on the unit sphere in $\mathfrak{Q}$. 
Abstract Wiener Theorem 1.5 (Gross [8]). If $\overline{\mathbb{Q}}$ is the completion of $\mathfrak{Q}$ in a measurable norm, then $\langle\mathfrak{Q} \mid \cdot\rangle$ defines a countably additive measure $\mu$ on $R(\overline{\mathfrak{Q}})$.

By standard measure theory $\mu$ extends to a (countably additive) probability measure on (the Borel field of) $\overline{\mathfrak{Q}}$. So the elements $f \in \mathscr{T}(\overline{\mathfrak{H}})$ extend trivially to measurable functions $f$ on $\overline{\mathfrak{Q}}$ so that

$$
\langle\mathfrak{Q} \mid f\rangle=\int_{\overline{\mathbb{Q}}} f\langle q\rangle d \mu\langle q\rangle .
$$

Since the identification of elements of $\overline{\mathfrak{H}}$ as measurable functions on $\overline{\mathfrak{Q}}$ is a densely defined bounded linear mapping from $\mathfrak{H}$ to $\mathscr{L}^{2}\left(\mu_{0}, \overline{\mathfrak{Q}}\right)$, it extends uniquely to all of $\mathfrak{H}$. Thus the elements of $f \in \mathscr{T}(\mathfrak{H})$ also extend to functions $f$ on $\overline{\mathbf{Q}}$ so that (1.10) holds.

Remark 1.6. Let $\mathfrak{Q}_{1}$ be a subspace of $\mathfrak{Q}$ and let $\overline{\mathfrak{Q}_{1}}$ be the completion of $\mathfrak{Q}_{1}$ in a measurable norm on $\mathfrak{Q}_{1}$. Let $\mu_{0}$ be the probability measure on $\overline{\mathfrak{Q}}_{1}$ defined from $\left\langle\mathfrak{Q}_{1} \mid \cdot\right\rangle$. Then for $f \in \mathscr{T}(\mathfrak{H})$

$$
\left\langle\mathfrak{Q}, \mathfrak{Q}_{1}, q_{1} \mid f\right\rangle=\int_{\mathbb{Q}_{1}} f\left\langle q_{1}+q\right\rangle d \mu_{0}\langle q\rangle .
$$

Remark 1.7. Suppose $\mathfrak{H}_{1}$ and $\mathfrak{Q}_{1}$ satisfy the conditions of Lemma 1.2. Then there are equivalent measures $\mu$ and $\mu_{q_{1}}$ on $\overline{\mathfrak{Q}}_{1}$ which are absolutely continuous with respect $\mu_{0}$ of (1.11) such that, for $f \in \mathscr{T}\left(\mathfrak{H}_{1}\right)$,

and

$$
\begin{aligned}
\langle\mathfrak{Q} \mid f\rangle & =\int_{\mathbb{Q}_{1}} f\langle q\rangle d \mu\langle q\rangle \\
\left\langle\mathfrak{Q}, \mathfrak{Q}_{1}, q_{1} \mid f\right\rangle & =\int_{\mathbb{Q}_{1}} f\langle q\rangle d \mu_{q_{1}}\langle q\rangle .
\end{aligned}
$$

\section{§ 2. The Free Markoff Field [5]}

If $\mathscr{R}$ is a closed set in $\mathbb{R}^{l+1}$, let

$\mathfrak{H}(\mathscr{R})=\left\{\phi \in \mathscr{S}^{\prime}\left(\mathbb{R}^{l+1}\right): \phi\right.$ real, supp $\left.\phi \subset \mathscr{R},\left\|\left(m^{2}-\Delta\right)^{-\frac{1}{2}} \phi\right\|_{2}<\infty\right\}$

where $\mathscr{S}^{\prime}$ is the Schwartz space of distributions and $\Delta$ is the Laplacian on $\mathscr{S}^{\prime}\left(\mathbb{R}^{l+1}\right), m>0 . \mathfrak{H}(\mathscr{R})$ is a Hilbert space in the indicated norm and it is a subspace of $\mathfrak{H}\left(\mathbb{R}^{l+1}\right)$. If $\mathscr{R}$ has a non-empty interior, let

$\mathfrak{Q}(\mathscr{R})$

$=\left\{\right.$ real Hilbert space in the norm $\left\|\left(m^{2}-\Delta\right)^{\frac{1}{2}} \cdot\right\|_{2}$ generated by $\left.\mathscr{C}_{0}^{\infty}(\mathscr{R})\right\}$

which is equal to $\mathscr{D}\left(\Delta_{\mathscr{R}}^{\frac{1}{2}}\right)$ in the norm $\left\|\left(m^{2}-\Delta_{\mathscr{R}}\right)^{\frac{1}{2}} \cdot\right\|_{2}$, where $\Delta_{\mathscr{R}}$ is the Dirichelet (or Friedrichs' expansion from $\mathscr{C}_{0}^{\infty}(\mathscr{R})$ ) Laplacian on $\mathscr{L}^{2}(\mathscr{R})$. $\mathfrak{Q}(\mathscr{R})$ is a subspace of $\mathfrak{Q}\left(\mathbb{R}^{l+1}\right)$. 
$\mathfrak{H}\left(\mathbb{R}^{l+1}\right)$ and $\mathfrak{Q}\left(\mathbb{R}^{l+1}\right)$ are a dual pair in an extension by continuity of the $\mathscr{L}^{2}\left(\mathbb{R}^{l+1}\right)$ inner product:

$$
\phi\langle q\rangle=\int_{\mathbb{R}^{i+1}} \phi(\boldsymbol{x}, t) q(\boldsymbol{x}, t) d \boldsymbol{x} d t .
$$

The Riesz identification is $\left(m^{2}-\Delta\right): \mathfrak{Q}\left(\mathbb{R}^{l+1}\right) \rightarrow \mathfrak{G}\left(\mathbb{R}^{l+1}\right)$. Also, if we treat $\mathfrak{H}(\mathscr{R})$ and $\mathfrak{Q}(\mathscr{R})$ as respective subspaces,

$$
\hat{Q}(\mathscr{R})^{\perp}=\mathfrak{H}\left(\mathscr{R}^{c}\right)
$$

for $\mathscr{R}$ open with $\mathscr{R}^{c}$ the complement in $\mathbb{R}^{l+1}, \hat{\mathfrak{Q}}(\mathscr{R})=\left(m^{2}-\Delta\right) \mathfrak{Q}(\mathscr{R})$. The free Markoff field on $\mathbb{R}^{l+1}$ is the identity mapping on $\mathfrak{H}\left(\mathbb{R}^{l+1}\right)$ which makes each element of the Hilbert space into a Gaussian random variable in the expectation $\left\langle\mathfrak{Q}\left(\mathbb{R}^{l+1}\right) \mid \cdot\right\rangle$. We will simply refer to $\left\langle\mathfrak{Q}\left(\mathbb{R}^{l+1}\right) \mid \cdot\right\rangle$ as the free field expectation and similarly to $\left\langle\mathbb{Q}\left(\mathbb{R}^{l+1}\right)\right.$, $\mathfrak{Q}(\mathscr{R})|\cdot\rangle$ as the Dirichelet free field expectation for the region $\mathscr{R}$.

Let $\mathscr{R} \subset \mathbb{R}^{l+1}$ be open with closure $\mathscr{R}$ and boundary $\partial \mathscr{R}$. Then Nelson's Markoff property is

$$
P_{\mathfrak{H}(\mathscr{R})} P_{\mathfrak{W}(\overline{\mathscr{R}})}=P_{\mathfrak{H}(\partial \mathscr{R})} P_{\mathfrak{H}(\overline{\mathscr{R}})}
$$

where $P_{\mathfrak{H}_{1}}$ denotes the orthogonal projection in $\mathfrak{H}\left(\mathbb{R}^{l+1}\right)$ to the subspace $\mathfrak{S}_{1}[5]$.

By (1.6) and (2.4), if $f \in \mathscr{T}\left(\mathfrak{H}\left(\mathbb{R}^{l+1}\right)\right)$ and $\mathscr{R} \subset \mathbb{R}^{l+1}$ is open,

$$
\left\langle\mathfrak{Q}\left(\mathbb{R}^{l+1}\right) \mid f\right\rangle=\left\langle\hat{\mathfrak{H}}\left(\mathscr{R}^{c}\right) \mid\left\langle\mathfrak{Q}\left(\mathbb{R}^{l+1}\right), \mathfrak{Q}(\mathscr{R}), \cdot \mid f\right\rangle\right\rangle .
$$

So, by (2.5) if $f \in \mathscr{T}(\mathfrak{H}(\overline{\mathscr{R}}))$,

$$
\left\langle\mathfrak{Q}\left(\mathbb{R}^{l+1}\right) \mid f\right\rangle=\left\langle\hat{\mathfrak{H}}(\partial \mathscr{R}) \mid\left\langle\mathfrak{Q}\left(\mathbb{R}^{l+1}\right), \mathfrak{Q}(\mathscr{R}), \mid f\right\rangle\right\rangle .
$$

If $\psi \in \mathfrak{H}(\partial \mathscr{R})$ we define Dirichelet free field expectation for the region $\mathscr{R}$ with boundary condition $\hat{\psi}$ to be $\left\langle\mathfrak{Q}\left(\mathbb{R}^{l+1}\right), \mathfrak{Q}(\mathscr{R}), \hat{\psi} \mid \cdot\right\rangle$. So $(2.7)$ gives the free field expectation as an average over the boundary conditions of the Dirichelet expectations.

Proposition 2.1. Let $\mathscr{R}_{1} \subset \mathscr{R}_{2} \subset \mathbb{R}^{l+1}$ with $\mathscr{R}_{1}$ closed, $\mathscr{R}_{2}$ open and $\partial \mathscr{R}_{1}$ and $\partial \mathscr{R}_{2}$ separated by a spherical shell. Then, restricted to $\mathscr{T}\left(\mathfrak{H}\left(\mathscr{R}_{1}\right)\right)$, the free field expectation $\left\langle\mathfrak{Q}\left(\mathbb{R}^{l+1}\right) \mid \cdot\right\rangle$ is equivalent to the Dirichelet free field expectation $\left\langle\mathfrak{Q}\left(\mathbb{R}^{l+1}\right), \mathfrak{Q}\left(\mathscr{R}_{2}\right), \hat{\psi} \mid \cdot\right\rangle, \psi \in \mathfrak{H}\left(\partial \mathscr{R}_{2}\right)$.

Proof. We will prove that the conditions (1.7) of Lemma 1.2 are satisfied with $\mathfrak{H}_{1}=\mathfrak{H}\left(\mathscr{R}_{1}\right)$ and $\mathfrak{Q}_{1}=\mathfrak{Q}\left(\mathscr{R}_{2}\right)$. From (2.4) and (2.5) we have that

$$
P_{\mathfrak{W}_{1}} P_{\mathfrak{Q}_{1}}^{\perp} P_{\mathfrak{W}_{1}}=P_{\mathfrak{S}\left(\partial \mathscr{R}_{1}\right)} P_{\mathfrak{W}\left(\partial \mathscr{R}_{2}\right)} P_{\mathfrak{W}\left(\partial \mathscr{R}_{1}\right)} P_{\mathfrak{W}\left(\mathscr{R}_{1}\right)}
$$

So we need to prove that this operator is Hilbert-Schmidt with norm less than one. By Lemma 1.3 and translation invariance it suffices to 
consider the case $\mathscr{R}_{1}=\left\{r \in \mathbb{R}^{l+1}:|r| \leqq R_{1}\right\}$ and $\left.\mathscr{R}_{2}=r \in \mathbb{R}^{l+1}:|r|<R_{2}\right\}$, $R_{1}<R_{2}$. Then we can explicitly diagonalize (2.8) by decomposing $\mathfrak{H}\left(\mathbb{R}^{l+1}\right)$ with spherical harmonics. For simplicity we write this out only for the $\mathbb{R}^{2}(l=1)$ case. Use polar coordinates $e^{i \theta} r \in \mathbb{R}^{2}$. If $\phi \in \mathfrak{H}\left(\mathbb{R}^{2}\right)$, $\phi\left(e^{i \theta} r\right)=\sum_{-\infty}^{\infty} e^{i n \theta} \phi_{n}(r)\left(\phi_{n}=\phi_{-n}\right.$ since $\mathfrak{H}\left(\mathbb{R}^{2}\right)$ is real) has Fourier transform $\sum_{-\infty}^{\infty} e^{i n \theta} \tilde{\phi}_{n}(r)$ where $\tilde{\phi}_{n}(r)=(-i)^{n} 2 \pi \int_{0}^{\infty} \phi_{n}\left(r^{\prime}\right) J_{n}\left(r r^{\prime}\right) r^{\prime} d r^{\prime} ;$ so $\left(\left(m^{2}-\Delta\right) \phi\right)_{n}(r)=\left(m^{2}+r^{2}\right) \tilde{\phi}_{\tilde{n}}(r)$ and in particular

$$
\left(\phi_{1}, \phi_{2}\right)_{\mathfrak{H}\left(\mathbb{R}^{2}\right)}=\sum_{n=-\infty}^{\infty} \int_{0}^{\infty} \frac{r}{m^{2}+r^{2}} \tilde{\phi}_{1,-n}(r) \tilde{\phi}_{2, n}(r) d r .
$$

This gives an orthogonal decomposition of $\mathfrak{H}\left(\mathbb{R}^{2}\right)$ that reduces the operators $P_{\mathfrak{S}\left(\partial \mathscr{R}_{i}\right)}$ and $P_{\mathfrak{S}\left(\mathscr{R}_{1}\right)}$ appearing in (2.8). Let $m=1$ and let

$$
\phi_{n, R}\left(e^{i \theta} r\right)=\frac{\cos n \theta}{2 \pi R \sqrt{I_{n}(R) K_{n}(R)}} \delta(r-R) .
$$

Since $\int_{0}^{\infty} \frac{r}{1+r^{2}} J_{n}\left(R_{1} r\right) J_{n}\left(R_{2} r\right) d r=I_{n}\left(R_{1}\right) K_{n}\left(R_{2}\right)$ for $R_{1} \leqq R_{2}$,

$$
\left(\phi_{n_{1}, R_{1}}, \phi_{n_{2}, R_{2}}\right)_{\mathfrak{G}\left(\mathbb{R}^{2}\right)}=\left\{\begin{array}{l}
0, \text { if } n_{1} \neq n_{2} \\
\left(\frac{I_{n}\left(R_{1}\right) K_{n}\left(R_{2}\right)}{K_{n}\left(R_{1}\right) I_{n}\left(R_{2}\right)}\right)^{\frac{1}{2}}, \quad \text { if } n_{1}=n=n_{2} .
\end{array}\right.
$$

So the $\phi_{n, R_{l}}$ form an orthonormal basis of $\mathfrak{H}\left(\partial \mathscr{R}_{i}\right)$ corresponding to the orthogonal decomposition given by (2.9). If $\phi \in \mathfrak{H}\left(\mathscr{R}_{1}\right)$, we can now compute:

$$
P_{\mathfrak{H}\left(\partial \mathscr{R}_{1}\right)} P_{\mathfrak{W}\left(\partial \mathscr{R}_{2}\right)} P_{\mathfrak{W}\left(\partial \mathscr{R}_{1}\right)} \phi=\sum_{n=0}^{\infty} \frac{I_{n}\left(R_{1}\right) K_{n}\left(R_{2}\right)}{K_{n}\left(R_{1}\right) I_{n}\left(R_{2}\right)}\left(\phi_{n, R_{1}}, \phi\right) \phi_{n, R_{1}} .
$$

So we need to show that

$$
\begin{gathered}
\frac{I_{n}\left(R_{1}\right) K_{n}\left(R_{2}\right)}{K_{n}\left(R_{1}\right) I_{n}\left(R_{2}\right)} \leqq \text { const. }<1 \\
\sum_{n=0}^{\infty}\left(\frac{I_{n}\left(R_{1}\right) K_{n}\left(R_{2}\right)}{K_{n}\left(R_{1}\right) I_{n}\left(R_{2}\right)}\right)^{2}<\infty .
\end{gathered}
$$

This can be seen from the representation

$$
I_{n}(R) K_{n}(S)=\frac{1}{2} \int_{\ln \left(\frac{S}{R}\right)}^{\infty} e^{-n t} J_{0}\left(\left\{2 R S \cosh t-R^{2}-S^{2}\right\}^{\frac{1}{2}}\right) d t
$$


for $R, S>0$ :

$$
\frac{I_{n}\left(R_{1}\right) K_{n}\left(R_{2}\right)}{K_{n}\left(R_{1}\right) I_{n}\left(R_{2}\right)}=\left\{1+\frac{\int_{\ln \left(\frac{R_{1}}{R_{2}}\right)}^{\ln \left(\frac{R_{2}}{R_{1}}\right)} e^{-n t} J_{0}\left(\{\}^{\frac{1}{2}}\right) d t}{\int_{\ln \left(\frac{R_{2}}{R_{1}}\right)}^{\infty} e^{-n t} J_{0}\left(\{\}^{\frac{1}{2}}\right) d t}\right\}^{-1}
$$

which is less than one and exponentially decreasing in $n$.

Proposition 2.2. If $q \in \mathbb{Q}\left(\mathbb{R}^{l+1}\right)$, then

$$
t \mapsto q(\cdot, t)
$$

is a continuous mapping from $\mathbb{R}$ to the Hilbert space $\mathscr{D}\left(\Delta^{\frac{1}{4}}\right)$ in $\left\|\left(m^{2}-\Delta_{l}\right)^{\frac{1}{4}} \cdot\right\|_{2}$ norm, where $\Delta_{l}$ is the Laplacian on $\mathbb{R}^{l}$. Similarly, if $\mathscr{R}=\boldsymbol{R}_{0} \times \mathbb{R}$ is an open rectangle in $\mathbb{R}^{l+1}$ and if $q \in \mathbb{Q}(\mathscr{R})$, then $(2.16)$ is also continuous with $\Delta_{l}$ replaced by the Dirichelet Laplacian for $\boldsymbol{R}_{0}$.

Proof. Let $\tilde{q}(\boldsymbol{p}, \omega)=\iint e^{i \boldsymbol{p} \cdot \boldsymbol{x}+i \omega \cdot t} q(\boldsymbol{x}, t) d \boldsymbol{x} d t$. Then

$$
\begin{aligned}
&\left\|\left(m^{2}-\Delta_{l}\right)^{\frac{1}{4}}\{q(\cdot, t)-q(\cdot, 0)\}\right\|_{2}^{2} \\
&=\frac{1}{(2 \pi)^{l}} \int\left(m^{2}+\boldsymbol{p}^{2}\right)^{\frac{1}{2}}\left|\frac{1}{2 \pi} \int\left\{e^{-i \omega t}-1\right\} \tilde{q}(\boldsymbol{p}, \omega) d \omega\right|^{2} d \boldsymbol{p} \\
& \leqq \frac{1}{(2 \pi)^{l+1}} \int\left(m^{2}+\boldsymbol{p}^{2}\right)^{\frac{1}{2}}\left\{\int \frac{1}{m^{2}+\boldsymbol{p}^{2}+\omega^{2}} d \omega\right\} \int\left(m^{2}+\boldsymbol{p}^{2}+\omega^{2}\right) \\
& \cdot\left|\left\{e^{-i \omega t}-1\right\} \tilde{q}(\boldsymbol{p}, \omega)\right|^{2} d \omega d \boldsymbol{p} \\
&= \frac{1}{2(2 \pi)^{l+1}} \iint\left(m^{2}+\boldsymbol{p}^{2}+\varepsilon^{2}\right)\left|\left\{e^{-i \omega t}-1\right\} \tilde{q}(\boldsymbol{p}, \omega)\right|^{2} d \omega d \boldsymbol{p}
\end{aligned}
$$

which goes to zero as $t \rightarrow 0$ by dominated convergence. In the Dirichelet case one replaces the $p$-Fourier transform by an expansion in the eigenvectors of the Dirichelet Laplacian. The same inequality and convergence then applies. (A similar inequality shows that $q(\cdot, t)$ is finite in $\left\|\left(m^{2}-\Delta_{l}\right)^{\frac{1}{4}} \cdot\right\|_{2}$ norm, etc. $)$

Remark 2.2. After the preparation of this paper, we received the preprint of Guerra, Rosen, and Simon [4] in which Proposition 2.1 is proved under the natural condition that $\partial R_{1}$ and $\partial R_{2}$ be bounded apart. (We keep the present formulation for the reader's convenience since the proof is completely elementary and explicit.)

\section{§ 3. Continuous Sample Paths}

Let $\mathscr{R}=\boldsymbol{R}_{0} \times(0, T)$ be a bounded open rectangle in $\mathbb{R}^{l+1}$. Let $\Delta_{0}$ be the Dirichelet Laplacian on $\boldsymbol{R}_{0}$. Let $\left\{\phi_{\alpha}\right\}$ be an orthonormal basis of $\mathscr{L}^{2}\left(\boldsymbol{R}_{0}\right)$ of eigenvectors of $\Delta_{0}$ and let $\left\{m_{\alpha}^{2}\right\}$ be the eigenvalues of $\left(m^{2}-\Delta_{0}\right)$ :

$$
\left(m^{2}-\Delta_{0}\right) \phi_{\alpha}=m_{\alpha}^{2} \phi_{\alpha} \text {. }
$$


If $q \in \mathscr{L}^{2}(\mathscr{R})$, let

$$
q_{\alpha}(t)=\int_{\boldsymbol{R}_{0}} \phi_{\alpha}(\boldsymbol{x}) q(\boldsymbol{x}, t) d \boldsymbol{x} .
$$

Theorem 3.1. The following norms are measurable on $\mathfrak{Q}(\mathscr{R})$ :

and

$$
\left.\begin{array}{l}
\sup _{0 \leqq t \leqq T}\left(\sum_{\alpha} \mid m_{\alpha}^{-\gamma} q_{\alpha}(t)^{p}\right)^{1 / p}, \quad 1 \leqq p<\infty, \quad \gamma>\frac{l}{p}-\frac{1}{2} \\
\sup _{0 \leqq t \leqq T} \sup _{\alpha}\left|m_{\alpha}^{-\gamma} q_{\alpha}(t)\right|, \quad \gamma>-\frac{1}{2}
\end{array}\right\}
$$

$\sup _{0 \leqq t \leqq T}\left\|\left(m^{2}-\Delta_{0}\right)^{-\frac{\gamma}{2}} q(\cdot, t)\right\|_{p^{\prime}}, \quad 2 \leqq p^{\prime} \leqq \infty, \quad \gamma>l\left(1-\frac{1}{p^{\prime}}\right)-\frac{1}{2}$.

In $\S 5$ we will prove measurability of the norm (3.3). That the norm (3.4) is also measurable follows because, by the Hausdorff, Young, and F. Riesz Theorem, the norm (3.4) is dominated by $M^{2 / p-1}$ times the norm (3.3), where $M=\sup \left|\phi_{\alpha}(x)\right|=2^{l}\left|\boldsymbol{R}_{0}\right|^{-\frac{1}{2}}$, if $\frac{1}{p}+\frac{1}{p^{\prime}}=1$. For simplicity of notation we shall refer everything that follows in this section to the norm (3.3); but, because of this domination, there are corresponding statements for the norm (3.4).

Let

$$
\mathfrak{Q}^{p, \gamma}(\mathscr{R})=\{\text { completion of } \mathfrak{Q}(\mathscr{R}) \text { in the norm (3.3)\}. }
$$

We can regard the elements $q \in \mathfrak{Q}^{p, \gamma}(\mathscr{R})$ as sample paths

$$
t \mapsto q(\cdot, t)
$$

which are continuous from $[0, T]$ in the norm

$$
\|\cdot\|_{p, \gamma} \equiv\left\{\begin{array}{l}
\left(\sum_{\alpha}\left|m_{\alpha}^{-\gamma}\left(\phi_{\alpha}, \cdot\right)_{2}\right|^{p}\right)^{1 / p}, \\
\sup _{\alpha}\left|m_{\alpha}^{-\gamma}\left(\phi_{\alpha}, \cdot\right)_{2}\right|, \quad p=\infty
\end{array}\right.
$$

for $1 \leqq p \leqq \infty, \gamma>\frac{l}{p}-\frac{1}{2}$. This continuity follows from the fact that $\mathscr{C}_{0}^{\infty}(\mathscr{R})$ is dense in $\mathfrak{Q}(\mathscr{R})$ and, by Lemma 1.4 and Theorem 3.1, the topology of $\mathfrak{Q}^{p, \gamma}(\mathscr{R})$ is weaker than that of $\mathfrak{Q}(\mathscr{R})$.

By Theorem 1.5 and Remark 1.6, we have the following:

Corollary 3.2. Let $1 \leqq p \leqq \infty, \gamma>\frac{l}{p}-\frac{1}{2} .\langle\mathfrak{Q}(\mathscr{R}) \mid \cdot\rangle$ defines $a$ probability measure $\mu_{0}$ on (the Borel field of) $\mathfrak{Q}^{p, \gamma}(\mathscr{R})$. The Dirichelet free field expectation of $f \in \mathscr{T}\left(\mathfrak{H}\left(\mathbb{R}^{l+1}\right)\right.$ is given by the integral

$$
\left\langle\mathbb{Q}\left(\mathbb{R}^{l+1}\right), \mathfrak{Q}(R), \hat{\psi} \mid f\right\rangle=\int_{\mathbb{Q}^{p, \gamma(R)}} f\langle\hat{\psi}+q\rangle d \mu_{0}\langle q\rangle .
$$


Let $\mathscr{R}_{1}$ be closed in $\mathscr{R}$ such that $\partial \mathscr{R}_{1}$ and $\partial \mathscr{R}$ are separated by a spherical shell. Then, by Remark 1.7 and Proposition 2.1, we have the following:

Corollary 3.3. Let $1 \leqq p \leqq \infty, \gamma>\frac{l}{p}-\frac{1}{2}$. The free field expectation of $f \in \mathscr{T}\left(\mathfrak{S}\left(\mathscr{R}_{1}\right)\right)$ is given by an integral

$$
\left\langle\mathfrak{Q}\left(\mathbb{R}^{l+1}\right) \mid f\right\rangle=\int_{\mathbb{Q}^{p, \nu}(\mathscr{R})} f\langle q\rangle d \mu\langle q\rangle .
$$

An equivalent measure exists to give the expectation (3.8) for $f \in \mathscr{T}\left(\mathfrak{H}\left(\mathscr{R}_{1}\right)\right)$. (By Remark 2.3, it suffices that $\partial \mathscr{R}_{1}$ and $\partial \mathscr{R}$ be bounded apart.)

\section{§ 4. Orthogonal Decomposition of $\mathfrak{Q}(R)$ and the O.U. Process}

Let $\mathscr{R}, \boldsymbol{R}_{0}, \Delta_{0},\left\{\phi_{\alpha}\right\}$ and $\left\{m_{\alpha}\right\}$ be as in $\S 3$. Let

and

$$
\mathfrak{Q}^{\alpha}(\mathscr{R})=\left\{\text { closed linear span of } \phi_{\alpha} \times q(t) \in \mathfrak{Q}(\mathscr{R})\right\}
$$

$$
\mathfrak{Q}^{m}\left(\left(T_{1}, T_{2}\right)\right)=\left\{q(t):\left\|\left(m^{2}-\frac{\partial^{2}}{\partial t^{2}}\right)^{\frac{1}{2}} q\right\|_{2}<\infty\right\},
$$

as a Hilbert space in the norm indicated, where $\frac{\partial^{2}}{\partial t^{2}}$ is the Dirichelet Laplacian on $\left[T_{1}, T_{2}\right]$. We have the isomorphism:

$$
\left\{\begin{array}{l}
\mathfrak{Q}^{\alpha}(\mathscr{R}) \approx \mathfrak{Q}^{m_{\alpha}}((0, T)) \\
\phi_{\alpha} \otimes q \sim q
\end{array}\right.
$$

and the orthogonal decomposition:

with $q_{\alpha}(t)$ given by (3.2).

$$
\left.\begin{array}{l}
\mathfrak{Q}(\mathscr{R})=\bigoplus_{\alpha} \mathbb{Q}^{\alpha}(\mathscr{R}) \\
q(\cdot, t)=\sum_{\alpha} \phi_{\alpha} \otimes q_{\alpha}(t)
\end{array}\right\}
$$

To prove Theorem 3.1, we have to establish the estimate (1.9) for the norm (3.3). We will do this in $\S 5$, using (4.4) to decompose the path (2.16) into statistically independent components. In the rest of the present section we will study the individual components, namely the O.U. process, for which we need an estimate on exit probabilities.

The $O . U$. process is the free Markoff field in one dimension, $l=0$; that is, it is given as the expectation $\left\langle\mathbb{Q}^{m}(\mathbb{R}) \mid \cdot\right\rangle$, in the notation (4.2). Note that the $\delta$-function, $\delta_{s}(t)=\delta(t-s)$, belongs to the dual $\mathfrak{S}^{m}(\mathbb{R})$ $=\mathfrak{Q}^{m}(\mathbb{R})$, and more generally that

$$
\left.\begin{array}{r}
\hat{\delta}_{s}(t) \\
\left(\delta_{t}, \delta_{s}\right)_{\mathfrak{S}^{m}(\mathbb{R})}
\end{array}\right\}=\frac{1}{2 \pi} \int_{-\infty}^{\infty} \frac{e^{-i \omega(t-s)}}{m^{2}+\omega^{2}} d \omega=\frac{1}{2 m} e^{-m|t-s|} .
$$


(Recall that denotes the Riesz identification of $\mathfrak{H}^{m}(\mathbb{R})$ with $\mathfrak{Q}^{m}(\mathbb{R})$.) So by (1.5), the $\delta_{t}$ are Gaussian random variables of mean zero and and covariance $\left\langle\mathfrak{Q}^{m}(\mathbb{R}) \mid \delta_{t} \delta_{s}\right\rangle=\frac{1}{\mathrm{~m}} \mathrm{e}^{-m|t-s|}$. Let $\left\{\hat{\delta}_{0}\right\}^{\perp}$ be the subspace of $\mathfrak{Q}^{m}(\mathbb{R})$ of elements orthogonal to $\hat{\delta}_{0}$; then the elements of $\mathbb{Q}^{m}(\mathbb{R})$ which take on the value $x$ at $t=0$ are of the form $q+2 m \times \hat{\delta}_{0}$ for $q \in\left\{\hat{\delta}_{0}\right\}^{\perp}$. The O.U. process, conditional by being tied to the value $x$ at $t=0$ is given by the expectation, in the notation (1.4),

$$
\left\langle\mathfrak{Q}^{m}(\mathbb{R}),\left\{\delta_{0}\right\}^{\perp}, 2 m x \hat{\delta}_{0} \mid \cdot\right\rangle .
$$

By (1.5), the $\delta_{t}$ are Gaussian in (4.6) with mean

and covariance

$$
\delta_{t}\left\langle 2 m x \hat{\delta}_{0}\right\rangle=2 m x\left(\delta_{0}, \delta_{t}\right)_{\mathfrak{Y}^{m}}=x e^{-m|t|}
$$

$$
\begin{aligned}
2\left(P_{\left\{\delta_{0^{+}}\right.} \delta_{t}, \delta_{s}\right) & =2\left(\delta_{t}, \delta_{s}\right)-2\left(\delta_{t}, \sqrt{2 m} \delta_{0}\right)\left(\sqrt{2 m} \delta_{0}, \delta_{s}\right) \\
& =\frac{1}{m} e^{-m|t-s|}-\frac{1}{m} e^{-m(|t|+|s|)} .
\end{aligned}
$$

In particular, since $\delta_{t}$ has mean (4.7) and covariance (4.8) with $s=t$, $\delta_{t}$ has the density in $y$

$$
P(x \mid y ; t)=\frac{1}{\sqrt{\pi}} \frac{1}{\sqrt{\frac{1}{m}\left(1-e^{-2 m|t|}\right)}} \exp \left\{-\frac{\left(y-x e^{-m|t|}\right)^{2}}{\frac{1}{m}\left(1-e^{-2 m|t|}\right)}\right\} .
$$

Of course, the O.U. process is well known, but we have presented the calculations (4.5)-(4.9) to illustrate the notation of $\S 1$. Denote the Laplace transform of $u(t)$ by $\tilde{u}(\lambda)=\int_{0}^{\infty} e^{-\lambda t} u(t) d t$. We will use the same notation for the two sided Laplace transform. Now, (4.9) has the Laplace transform in the case $m=1$ (cf. [16]).

$$
\tilde{P}(x \mid y ; \lambda)=\frac{2^{\lambda}}{\sqrt{\pi}} \Gamma(\lambda) H_{-\lambda}(-x) H_{-\lambda}(y), \quad x \leqq y,
$$

where $H_{-\lambda}(z)$ is the Hermite function of degree $-\lambda$,

solving the equation

$$
H_{-\lambda}(z)=\frac{1}{\Gamma(\lambda)} \int_{0}^{\infty} e^{-t^{2}-2 t z} t^{\lambda-1} d t,
$$

$$
\left\{\frac{1}{2} \frac{\partial^{2}}{\partial z^{2}}-z \frac{\partial}{\partial z}\right\} H_{-\lambda}(z)=\lambda H_{-\lambda}(z) .
$$

Theorem 3.1 and its corollaries are illustrated in the $l=0$ case (the O.U. process) by the classical Wiener Theorem which says, in the language of Gross [8], that the norm

$$
\sup _{0 \leqq t \leqq T}|q(t)|
$$


is measurable on $\mathfrak{Q}^{m}([0, T])$, giving the result as in (3.6) that the paths $t \mapsto q(t)$ are continuous, $q(0)=0=q(T)$.

Let $\operatorname{Prob}\{\}$ be defined from $\left\langle\mathfrak{Q}^{m}([0, T]) \mid \cdot\right\rangle$ as in (1.9). We need an estimate on the probability that the norm (4.13) is greater than $a$ which is strong in a limit $m \rightarrow \infty, a \rightarrow 0$, corresponding to the fact that we need to control infinitely many components in (4.4). Let

$$
F_{a, T}\langle q\rangle= \begin{cases}1, & \text { if } q(t) \geqq a, \quad \text { some } t \in(0, T) \\ 0, & \text { otherwise } .\end{cases}
$$

We can approximate $F_{a, T}$ by tame functions on $\mathfrak{Q}^{m}([0, T])$ by restricting $t$ in (4.14) to finite sets and using the path continuity; so $F_{a, T}$ has appropriate measurability properties, etc. (by standard methods). By symmetry,

$$
\operatorname{Prob}\left\{\sup _{0 \leqq t \leqq T}|q(t)| \geqq a\right\} \leqq 2\left\langle\mathfrak{Q}^{m}((0, T)) \mid F_{a, T}\right\rangle .
$$

Lemma 4.1. There are positive constants independent of $m, a, T$ so that

$$
\left\langle\mathfrak{Q}^{m}((0, T)) \mid F_{a, T}\right\rangle \leqq \text { const. }[m T+1] e^{-c m a^{2}} .
$$

The square bracket denotes the integral part of $m T+1$; but this coefficient is not of significance. The constants will be obtained in (4.26) and (4.31). The scale transformation $q(t) \mapsto m q\left(\frac{t}{m}\right)$ is a unitary mapping from $\mathfrak{Q}^{m}((0, T))$ to $\mathfrak{Q}^{1}((0, m T))$. In particular, $\left\langle\mathfrak{Q}^{m}((0, T)) \mid F_{a, T}\right\rangle$ $=\left\langle\mathfrak{Q}^{1}((0, m T)) \mid F_{\sqrt{m} a, m T}\right\rangle$. So (4.16) is equivalent to

$$
\left\langle\mathbb{Q}_{1}((0, T)) \mid F_{a, T}\right\rangle \leqq \text { const. }[T+1] e^{-c a^{2}} .
$$

For simplicity we will begin with the case of the O.U. process tied only at the origin, proving the corresponding inequality

$$
\left\langle\mathfrak{Q}^{1}(\mathbb{R}),\left\{\hat{\delta}_{0}\right\}^{\perp} \mid F_{a, T}\right\rangle \leqq[T+1] e^{-c a^{2}} .
$$

The general techniques are found in Feller [16].

Proof. If $f_{a}(x, t) d t$ is the probability of reaching a for the first time at time $t$ having started at $x$ at time zero and $x<a<y$, then (by (4.9) with $m=1$ )

$$
P(x \mid y ; t)=\int_{0}^{t} f_{a}\left(x ; t^{\prime}\right) P\left(a \mid y ; t-t^{\prime}\right) d t^{\prime} .
$$

So, under the Laplace transform

$$
\tilde{f_{a}}(x ; \lambda)=\frac{\tilde{P}(x \mid y ; \lambda)}{\tilde{P}(a \mid y ; \lambda)}=\frac{H_{-\lambda}(-x)}{H_{-\lambda}(a)},
$$


by (4.10). We observe that $H_{-\lambda}(-z)$ is the two-sided Laplace transform of the function

$$
\begin{aligned}
g(z, t) & =\exp \left\{-e^{-2 t}+2 z e^{-t}\right\}: \\
H_{-\lambda}(-z) & =\frac{1}{\Gamma(\lambda)} \tilde{g}(z, \lambda), \quad \lambda>0 .
\end{aligned}
$$

From (4.20) and (4.22) we have that

$$
g(x, t)=\int_{0}^{\infty} f_{a}\left(x ; t^{\prime}\right) g\left(a, t-t^{\prime}\right) d t^{\prime} .
$$

Since for all $t, g(0, t) \leqq 1$,

$$
\int_{0}^{\infty} f_{a}\left(0 ; t^{\prime}\right) g\left(a, t-t^{\prime}\right) d t^{\prime} \leqq 1 .
$$

Since $f_{a}$ and $g$ are positive functions and since

with

$$
g(a, t) \geqq e^{c a^{2}}, \quad-\ln a \leqq t \leqq 1-\ln a,
$$

$$
c=2 e^{-1}-e^{-2}>0 .
$$

It follows from (4.24) that

$$
\int_{\tau}^{\tau+1} f_{a}(0 ; t) d t \leqq e^{-c a^{2}}
$$

for all $\tau \geqq 0$. Since, by the continuity of the paths,

$$
\left\langle\mathbb{Q}^{1}(\mathbb{R}),\left\{\tilde{\delta}_{0}\right\}^{\perp} \mid F_{a, T}\right\rangle=\int_{0}^{T} f_{a}(0 ; t) d t
$$

we obtain from (4.27) the inequality (4.18).

Now to prove (4.17), let $R_{a}(x \mid y ; t) d y$ be the probability of reaching $y$ at time $t$, having started at $x$ without having reached $a$. Then, $x, y<a$,

So

$$
P(x \mid y ; T)=R_{a}(x \mid y ; T)+\int_{0}^{T} f_{a}(x ; t) P(a \mid y ; T-t) d t .
$$

$$
\begin{aligned}
\left\langle\mathfrak{Q}^{1}((0, T)) \mid F_{a, T}\right\rangle & =1-\frac{R_{a}(0 \mid 0 ; T)}{P(0 \mid 0 ; T)} \\
& =\int_{0}^{T} \frac{P(a \mid 0 ; T-t)}{P(0 \mid 0 ; T)} f_{a}(x ; t) d t .
\end{aligned}
$$

We obtain (4.17) from (4.27), (4.30) and the inequality

$$
\frac{P(a \mid 0 ; T-t)}{P(0 \mid 0 ; T)}=\left(\frac{1-e^{-2 T}}{1-e^{-2(T-t)}}\right)^{\frac{1}{2}} e^{-\frac{a^{2} e^{-2(T-t)}}{1-e^{-2(T-t)}}} \leqq \text { const. },
$$

(provided $a$ is bounded away from the origin). 


\section{$\S 5$. Proof of Measurability}

We now prove Theorem 3.1, using the orthogonal decomposition (4.4) and Lemma 4.1. We have to establish the inequality (1.9) for the norm (3.3).

In the eigenvalue Eq. (3.1) we can use the index $\alpha=\left(\alpha_{1}, \ldots, \alpha_{l}\right) \in \mathbb{Z}_{+}^{l}$ with

$$
m_{\alpha}^{2}-m^{2}=\text { const. } \sum_{i=1}^{l} \alpha_{i}^{2} \text {. }
$$

So we have the convergence

$$
\sum_{\alpha} m_{\alpha}^{-l-\varepsilon}<\infty, \quad \varepsilon>0 .
$$
Suppose that $1 \leqq p<\infty$. The condition on $\gamma$ is $\gamma>\frac{l}{p}-\frac{1}{2}$; so we can
choose $\varepsilon>0$ so that

$$
\beta=\gamma-\frac{l+\varepsilon}{p}>-\frac{1}{2} \text {. }
$$

Given $a>0$, as in (1.9), we remove a finite part $I_{f}$ of the index set so that the infinite part $I_{i}=\mathbb{Z}_{+}^{l}-I_{f}$ has the following properties:

$$
\begin{gathered}
\prod_{\alpha \in I_{i}}\left\{1-2\left\langle Q^{m_{\alpha}}((0, T)) \mid F_{m \beta}, T\right\rangle\right\} \geqq \sqrt{1-a} \\
\left(\sum_{\alpha \in l_{l}} m_{\alpha}^{-l-\varepsilon}\right)^{1 / p}<\frac{a}{2} .
\end{gathered}
$$

That (5.4) is possible follows from Lemma 4.1 and (5.3) while (5.5) is possible by (5.2).

Let Prob \{\} be defined on the cylinder sets of $\mathfrak{Q}(\mathscr{R})$ from $\langle\mathfrak{Q}(\mathscr{R}) \mid \cdot\rangle$ and let $\operatorname{Prob}_{m}\{\}$ be defined from $\left\langle\mathfrak{Q}^{m}((0, T)) \mid \cdot\right\rangle$ on the space of continuous paths. Write

$$
\mathfrak{Q}_{i}=\bigoplus_{\alpha \in I i} \mathfrak{Q}^{\alpha}(\mathscr{R}) \text { and } \mathfrak{Q}_{f}=\bigoplus_{\alpha \in l_{f}} \mathfrak{Q}^{\alpha}(\mathscr{R}) .
$$

If $P_{i}$ is a finite dimensional orthogonal projection in $\mathfrak{Q}_{i}$, then, in the notation (3.7),

$$
\begin{aligned}
& \operatorname{Prob}\left\{\left\|P_{i} q(\cdot, t)\right\|_{p, \gamma} \leqq \frac{a}{2}, \quad \text { all } t \in[0, T]\right\} \\
& \geqq \operatorname{Prob}\left\{\left.\sum_{\alpha} m_{\alpha}^{-\gamma p}\left(P_{i} q\right)_{\alpha}(t)\right|^{p} \leqq \sum_{\alpha \in I_{l}} m_{\alpha}^{-l-\varepsilon}, \quad t \in[0, T]\right\} \\
& \geqq \prod_{\alpha \in I_{l}} \operatorname{Prob}_{m_{\alpha}}\left\{\left|q_{\alpha}(t)\right| \leqq m_{\alpha}^{\gamma-\frac{l+\varepsilon}{p}}, \quad t \in[0, T]\right\} \\
& \geqq \prod_{\alpha \in I_{i}}\left\{1-2\left\langle\mathfrak{Q}^{m_{\alpha}}((0, T)) \mid F_{m_{k}, T}\right\rangle\right\} \geqq \sqrt{1-a} .
\end{aligned}
$$

The last two inequalities are just (4.15) and (5.4); the first follows from (5.5). The second follows via the orthogonal decomposition (4.4) and the isomorphism (4.3) and the fact that the restriction $P_{i}$ decreases exit probabilities. An uninteresting technicality arises because $\operatorname{Prob}\{\}$ 
is only defined on cylinder sets; so to remove $P_{i}$ in establishing the second inequality we can introduce finite dimensional projections $P_{i}^{(\alpha)}$ in $\mathfrak{Q}^{\alpha}(\mathscr{R})$ such that $P_{i}=\sum_{\alpha \in I_{i}} P_{i}^{(\alpha)} P_{i}$ and use approximations that involve finite subsets of $I_{i}$. If $p=\infty$, the overall inequality holds by a similar argument.

On the other hand, we will find a finite dimensional subspace $\mathfrak{Q}_{a}$ in $Q_{f}$ such that

$$
\operatorname{Prob}\left\{\left\|P_{f} q(\cdot, t)\right\|_{p, \gamma} \leqq \frac{a}{2}, \quad t \in[0, T]\right\} \geqq \sqrt{1-a},
$$

for all finite dimensional projections $P_{f}$ in $\mathfrak{Q}_{f}$ orthogonal to $\mathfrak{Q}_{a}$. So, if $P$ is an arbitrary finite dimensional projection in $\mathbb{Q}(\mathscr{R})=\mathfrak{Q}_{f} \oplus \mathfrak{Q}_{i}$ that is orthogonal to $\mathfrak{Q}_{a}$ and $P_{i}$ and $P_{f}$ are projections as in (5.7) and (5.8) such that $P=P_{i} P+P_{f} P$,

$$
\begin{aligned}
& \operatorname{Prob}\left\{\|P q(\cdot, t)\|_{p, \gamma} \leqq a, \quad t \in[0, T]\right\} \\
\geqq & \operatorname{Prob}\left\{\left\|P_{i} q(\cdot, t)\right\|_{p, \gamma} \leqq \frac{a}{2}, \quad\left\|P_{f} q(\cdot, t)\right\|_{p, \gamma} \leqq \frac{a}{2}, \quad t \in[0, T]\right\}(5.9 \\
\geqq & 1-a .
\end{aligned}
$$

This is the desired inequality (1.9) for the norm (3.3).

To complete the proof we have to find $\mathfrak{Q}_{a}$ in $\mathfrak{Q}_{f}$ so that (5.8) holds. It suffices to consider the $\alpha \in I_{f}$ components separately. That is, it suffices to consider sufficiently small $\delta_{\alpha}>0$ and find finite dimensional subspaces $\mathfrak{Q}_{a}^{\alpha}$ in $\mathfrak{Q}^{m_{\alpha}}((0, T))$ such that, for all orthogonal projections $P^{(\alpha)}$ in $\mathfrak{Q}^{m_{\alpha}}((0, T))$ orthogonal to $\mathfrak{Q}_{a}^{\alpha}$,

$$
\operatorname{Prob}_{m_{\alpha}}\left\{\left|P^{(\alpha)} q_{\alpha}(t)\right| \leqq \delta_{\alpha}, t \in[0, T]\right\} \geqq(1-a)^{\frac{1}{2 \# I_{f}}}
$$

We can set $Q_{a}^{\alpha}=\left\{\hat{\delta}_{t_{i}}\right\}$, the space spanned by $\hat{\delta}_{t_{i}}$ for $0<t_{1}<t_{2}<\cdots<t_{n}<T$ with $t_{i}-t_{j-1}$ chosen sufficiently small. That this is sufficient follows from (4.15) and the estimate

$$
\left\langle Q^{m}\left(\left(0, T_{1}\right)\right) \mid F_{a, T_{1}}\right\rangle \leqq \text { const. } e^{- \text {const. } a^{2} T_{1}^{-1}},
$$

where the constants are independent of $m$. (5.11) is a well known estimate on the exit probabilities of the Wiener process, case $m=0$ (cf. Ray [17]). Since the norm in $Q^{m}\left(\left(0, T_{1}\right)\right)$ increases as $m$ increases, (5.11) holds for all $m$.

\section{References}

1. Nelson,E.: Quantum fields and Markoff fields, in Proceedings of Summer Institute of Partial Differential Equations, Berkeley 1971. Amer. Math. Soc., Providence, 1973

2. Albeverio, S., Hoegh-Krohn, R.: Uniqueness of the physical vacuum and the Wightman functions in the infinite volume limit for some non-polynomial interactions. Commun. math. Phys., to appear

3. Feldman, J.: A relativistic Feynman-Kac formula. Nuclear Phys., to appear 
4. Guerra, F., Rosen, L., Simon, B.: The $P(\phi)_{2}$ Euclidean quantum field theory as classical statistical mechanics. Princeton preprint

5. Nelson, E.: J. Funct. Anal. 12, $211-227$ (1973)

6. Varadhan,S.R.S.: Stochastic Processes, Lecture Notes of Courant Institute of Mathematical Sciences, N.Y.U.

7. Gel'fand,I.M., Vilenkin, N. Ya.: Generalized Functions, Volume 4, New York and London: Academic Press 1964

8. Gross, L.: Abstract Wiener space, in Fifth Berkeley Sympos. Math. Statist. and Probability. Berkeley: University of California Press 1967

9. Dimock, J., Glimm, J.: Measures on the Schwartz distribution space and applications to $P(\phi)_{2}$ field theories, N.Y.U. preprint

10. Segal, I.: Trans. Amer. Math. Soc. 88, $12-41$ (1958)

11. Glimm, J.: Commun. math. Phys. 8, 12-25 (1968)

12. Halmos, P.R.: Measure Theory. Princeton: D. van Nostrand Co. Inc., 1950

13. Rosen, L.: Commun. math. Phys. 16, 157-183 (1970)

14. Newman, C.: The construction of stationary two-dimensional Markoff fields with applications to quantum field theory, N.Y.U. preprint

15. Gross, L.: Trans. Amer. Math. Soc. 105, 372-390 (1962)

16. Feller, W.: An Introduction to Probability Theory and its Applications Volume II. New York, London, Sydney: John Wiley \& Sons, Inc., 1966

17. Ray, D.: Trans. Amer. Math. Soc. 77, 299-321 (1954)

\author{
J. T. Cannon \\ Mathematics Department \\ Rockefeller University \\ York Ave and 66th Street \\ New York, N.Y. 10021, USA
}


